\title{
Multiplicity Results Using Bifurcation Techniques for a Class of Fourth-Order $m$-Point Boundary Value Problems
}

\author{
Yansheng Liu' ${ }^{1}$ and Donal O'Regan ${ }^{2}$ \\ ${ }^{1}$ Department of Mathematics, Shandong Normal University, Jinan 250014, China \\ ${ }^{2}$ Department of Mathematics, National University of Ireland, Galway, Ireland
}

Correspondence should be addressed to Yansheng Liu, yanshliu@gmail.com

Received 13 March 2009; Accepted 12 April 2009

Recommended by Juan J. Nieto

By using bifurcation techniques, this paper investigates the existence of nodal solutions for a class of fourth-order $m$-point boundary value problems. Our results improve those in the literature.

Copyright (C) 2009 Y. Liu and D. O'Regan. This is an open access article distributed under the Creative Commons Attribution License, which permits unrestricted use, distribution, and reproduction in any medium, provided the original work is properly cited.

\section{Introduction}

Consider the following fourth order $m$-point boundary value problem (BVP, for short)

$$
\begin{gathered}
u^{(4)}(t)=f\left(u(t), u^{\prime \prime}(t)\right), \quad t \in(0,1) \\
u^{\prime}(0)=0, \quad u(1)=\sum_{i=1}^{m-2} \alpha_{i} u\left(\eta_{i}\right) \\
u^{\prime \prime \prime}(0)=0, \quad u^{\prime \prime}(1)=\sum_{i=1}^{m-2} \alpha_{i} u^{\prime \prime}\left(\eta_{i}\right),
\end{gathered}
$$

where $f: \mathbb{R} \times \mathbb{R} \rightarrow \mathbb{R}$ is a given sign-changing continuous function, $m \geq 3, \eta_{i} \in(0,1)$, and $\alpha_{i}>0$ for $i=1, \ldots, m-2$ with

$$
\sum_{i=1}^{m-2} \alpha_{i}<1
$$


Multi-point boundary value problems for ordinary differential equations arise in different areas of applied mathematics and physics. The existence of solutions of the second order multi-point boundary value problems has been studied by many authors and the methods used are the nonlinear alternative of Leray-Schauder, coincidence degree theory, fixed point theorems in cones and global bifurcation techniques (see [1-9], and the references therein). In [5], Ma investigated the existence and multiplicity of nodal solutions for

$$
\begin{aligned}
& u^{\prime \prime}(t)+f(u(t))=0, \quad t \in(0,1) \\
& u^{\prime}(0)=0, \quad u(1)=\sum_{i=1}^{m-2} \alpha_{i} u\left(\eta_{i}\right)
\end{aligned}
$$

when

$$
\eta_{i} \in \mathbb{Q}(i=1,2, \ldots, m-2) \quad \text { with } 0<\eta_{1}<\eta_{2}<\cdots<\eta_{m-2}<1,
$$

and $\alpha_{i}>0$ for $i=1, \ldots, m-2$ satisfying (1.2). He obtained some results on the spectrum of the linear operator corresponding to (1.1). It should be pointed out that the main tool used in [5] is results on bifurcation coming from the trivial solutions and we note no use was made of global results on bifurcation from infinity.

Recently [10] Wei and Pang studied the existence and multiplicity of nontrivial solutions for the fourth order $m$-point boundary value problems:

$$
\begin{gathered}
u^{(4)}(t)=f\left(u(t), u^{\prime \prime}(t)\right), \quad t \in(0,1) \\
u(0)=0, \quad u(1)=\sum_{i=1}^{m-2} \alpha_{i} u\left(\eta_{i}\right) \\
u^{\prime \prime}(0)=0, \quad u^{\prime \prime}(1)=\sum_{i=1}^{m-2} \alpha_{i} u^{\prime \prime}\left(\eta_{i}\right),
\end{gathered}
$$

where $f: \mathbb{R} \times \mathbb{R} \rightarrow \mathbb{R}$ is a given sign-changing continuous function, $m \geq 3, \eta_{i} \in(0,1)$, and $\alpha_{i}>0$ for $i=1, \ldots, m-2$ satisfies (1.2).

Motivated by $[5,10]$, in this paper we consider the existence and multiplicity of nodal solutions for BVP (1.1). The method used here is Rabinowitz's global bifurcation theorem. To the best of our best knowledge, only [10] seems to have considered the existence of nontrivial or positive solutions of the nonlinear multi-point boundary value problems for fourth order differential equations. As in $[5,10]$ we suppose (1.2) is satisfied throughout.

The paper is organized as follows. Section 2 gives some preliminaries. Section 3 is devoted to the existence of multiple solutions for BVP (1.1). To conclude this section we give some notation and state three lemmas, which will be used in Section 3. Following the notation of Rabinowitz, let $E$ be a real Banach space and $L: E \rightarrow E$ be a compact linear map. If there exists $\mu \in \mathbb{R}=[0,+\infty)$ and $0 \neq v \in E$ such that $v=\mu L v, \mu$ is said to be a real characteristic 
value of $L$. The set of real characteristic values of $L$ will be denoted by $\sigma(L)$. The multiplicity of $\mu \in \sigma(L)$ is

$$
\operatorname{dim} \bigcup_{j=1}^{\infty} N\left((I-\mu L)^{j}\right)
$$

where $N(A)$ denotes the null space of $A$. Suppose that $H: \mathbb{R} \times E \rightarrow E$ is compact and $H(\lambda, u)=o(\|u\|)$ at $u=0$ uniformly on bounded $\lambda$ intervals. Then

$$
u=\lambda L u+H(\lambda, u)
$$

possesses the line of trivial solutions $\Theta=\{(\lambda, 0) \mid \lambda \in \mathbb{R}\}$. It is well known that if $\mu \in \mathbb{R}$, a necessary condition for $(\mu, 0)$ to be a bifurcation point of (1.7) with respect to $\Theta$ is that $\mu \in$ $\sigma(L)$. If $\mu$ is a simple characteristic value of $L$, let $v$ denote the eigenvector of $L$ corresponding to $\mu$ normalized so $\|v\|=1$. By $\Sigma$ we denote the closure of the set of nontrivial solutions of (1.7). A component of $\Sigma$ is a maximal closed connected subset. It was shown in (Rabinowitz $[11$, Theorems 1.3, 1.25, 1.27]), the following.

Lemma 1.1. If $\mu \in \sigma(L)$ is simple, then $\Sigma$ contains a component $C_{\mu}$ which can be decomposed into two subcontinua $C_{\mu^{\prime}}^{+} C_{\mu}^{-}$such that for some neighborhood B of $(\mu, 0)$,

$$
(\lambda, u) \in C_{\mu}^{+}\left(C_{\mu}^{-}\right) \cap B, \quad(\lambda, u) \neq(\mu, 0)
$$

implies $(\lambda, u)=(\lambda, \alpha v+w)$ where $\alpha>0(\alpha<0)$ and $|\lambda-\mu|=o(1),\|w\|=o(|\alpha|)$ at $\alpha=0$.

Moreover, each of $C_{\mu}^{+}, C_{\mu}^{-}$either

(i) meets infinity in $\Sigma$, or

(ii) meets $(\widehat{\mu}, 0)$ where $\mu \neq \hat{\mu} \in \sigma(L)$, or

(iii) contains a pair of points $(\lambda, u),(\lambda,-u), u \neq 0$.

The following are global results for (1.7) on bifurcation from infinity (see, Rabinowitz [9, Theorem 1.6 and Corollary 1.8]).

Lemma 1.2. Suppose $L$ is compact and linear, $H(\lambda, u)$ is continuous on $\mathbb{R} \times E, H(\lambda, u)=o(\|u\|)$ at $u=\infty$ uniformly on bounded $\lambda$ intervals, and $\|u\|^{2} H\left(\lambda, u /\|u\|^{2}\right)$ is compact. If $\mu \in \sigma(L)$ is of odd multiplicity, then $\Sigma$ possesses an unbounded component $D_{\mu}$ which meets $(\mu, \infty)$. Moreover if $\Lambda \subset \mathbb{R}$ is an interval such that $\Lambda \cap \sigma(L)=\{\mu\}$ and $\wp$ is a neighborhood of $(\mu, \infty)$ whose projection on $\mathbb{R}$ lies in $\Lambda$ and whose projection on $E$ is bounded away from 0 , then either

(i) $D_{\mu} \backslash \wp$ is bounded in $\mathbb{R} \times E$ in which case $D_{\mu} \backslash \wp$ meets $\Theta=\{(\lambda, 0) \mid \lambda \in \mathbb{R}\}$ or

(ii) $D_{\mu} \backslash \wp$ is unbounded.

If (ii) occurs and $D_{\mu} \backslash \mathfrak{\wp}$ has a bounded projection on $\mathbb{R}$, then $D_{\mu} \backslash \mathfrak{\wp}$ meets $(\widehat{\mu}, \infty)$ where $\mu \neq \widehat{\mu} \in \sigma(L)$.

Lemma 1.3. Suppose the assumptions of Lemma 1.2 hold. If $\mu \in \sigma(L)$ is simple, then $D_{\mu}$ can be decomposed into two subcontinua $D_{\mu}^{+}, D_{\mu}^{-}$and there exists a neighborhood $\mathfrak{I} \subset \wp$ of $(\mu, \infty)$ such that $(\lambda, u) \in D_{\mu}^{+}\left(D_{\mu}^{-}\right) \cap \mathfrak{I}$ and $(\lambda, u) \neq(\mu, \infty)$ implies $(\lambda, u)=(\lambda, \alpha v+w)$ where $\alpha>0(\alpha<0)$ and $|\lambda-\mu|=o(1),\|w\|=o(|\alpha|)$ at $|\alpha|=\infty$. 


\section{Preliminaries}

Let $X=C[0,1]$ with the norm $\|u\|=\max _{t \in[0,1]}|u(t)|, Y=\left\{u \in C^{1}[0,1]: u^{\prime}(0)=0, u(1)=\right.$ $\left.\sum_{i=1}^{m-2} \alpha_{i} u\left(\eta_{i}\right)\right\}$ with the norm $\|u\|_{1}=\max \left\{\|u\|,\left\|u^{\prime}\right\|\right\}, Z=\left\{u \in C^{2}[0,1]: u^{\prime}(0)=0, u(1)=\right.$ $\left.\sum_{i=1}^{m-2} \alpha_{i} u\left(\eta_{i}\right)\right\}$ with the norm $\|u\|_{2}=\max \left\{\|u\|,\left\|u^{\prime}\right\|,\left\|u^{\prime \prime}\right\|\right\}$. Then $X, Y$, and $Z$ are Banach spaces.

For any $C^{1}$ function $u$, if $u\left(t_{0}\right)=0$, then $t_{0}$ is a simple zero of $u$ if $u^{\prime}\left(t_{0}\right) \neq 0$. For any integer $k \in \mathbb{N}$ and any $v \in\{ \pm\}$, as in [6], define sets $T_{k}^{v} \subset Z$ consisting of the set of functions $u \in Z$ satisfying the following conditions:

(i) $u^{\prime}(0)=0, v u(0)>0$ and $u^{\prime}(1) \neq 0$;

(ii) $u^{\prime}$ has only simple zeros in $(0,1)$, and has exactly $k-1$ such zeros;

(iii) $u$ has a zero strictly between each two consecutive zeros of $u^{\prime}$.

Note $T_{k}^{-}=-T_{k}^{+}$and let $T_{k}=T_{k}^{-} \cup T_{k}^{+}$. It is easy to see that the sets $T_{k}^{-}$and $T_{k}^{+}$are disjoint and open in $Z$. Moreover, if $u \in T_{k}^{v}$, then $u$ has at least $k-2$ zeros in $(0,1)$, and at most $k-1$ zeros in $(0,1]$.

Let $E=\mathbb{R} \times Y$ under the product topology. As in [12], we add the points $\{(\lambda, \infty): \lambda \in \mathbb{R}\}$ to the space $E$. Let $\Phi_{k}^{+}=\mathbb{R} \times T_{k}^{+}, \Phi_{k}^{-}=\mathbb{R} \times T_{k}^{-}$, and $\Phi_{k}=\mathbb{R} \times T_{k}$.

We first convert BVP (1.1) into another form. Suppose $u(t)$ is a solution of BVP (1.1). Let $v(t)=-u^{\prime \prime}(t)$. Notice that

$$
\begin{gathered}
u^{\prime \prime}(t)+v(t)=0, \quad t \in I \\
u^{\prime}(0)=0, \quad u(1)=\sum_{i=1}^{m-2} \alpha_{i} u\left(\eta_{i}\right) .
\end{gathered}
$$

Thus $u(t)$ can be written as

$$
u(t)=L v(t)
$$

where the operator $L$ is defined by

$$
L v(t):=\int_{0}^{1} H(t, s) v(s) d s, \quad \forall v \in Y,
$$

where

$$
\begin{aligned}
& H(t, s)=G(t, s)+\frac{\sum_{i=1}^{m-2} \alpha_{i} G\left(\eta_{i}, s\right)}{1-\sum_{i=1}^{m-2} \alpha_{i} \eta_{i}}, \\
& G(t, s)= \begin{cases}1-t, & 0 \leq s \leq t \leq 1 ; \\
1-s, & 0 \leq t \leq s \leq 1 .\end{cases}
\end{aligned}
$$


Therefore we obtain the following equivalent form of (1.1)

$$
\begin{gathered}
v^{\prime \prime}(t)+f((L v)(t),-v(t))=0, \quad t \in(0,1) \\
v^{\prime}(0)=0, \quad v(1)=\sum_{i=1}^{m-2} \alpha_{i} v\left(\eta_{i}\right) .
\end{gathered}
$$

For the rest of this paper we always suppose that the initial value problem

$$
\begin{gathered}
v^{\prime \prime}(t)+f((L v)(t),-v(t))=0, \quad t \in(0,1) ; \\
v\left(t_{0}\right)=v^{\prime}\left(t_{0}\right)=0
\end{gathered}
$$

has the unique trivial solution $v \equiv 0$ on $[0,1]$ for any $t_{0} \in[0,1]$; in fact some suitable conditions such as a Lipschitz assumption or $f \in C^{1}$ guarantee this.

Define two operators on $Y$ by

$$
(A v)(t):=(L F v)(t), \quad(F v)(t):=f((L v)(t),-v(t)), \quad t \in I, v \in Y
$$

Then it is easy to see the following lemma holds.

Lemma 2.1. The linear operator $L$ and operator A are both completely continuous from $Y$ to $Y$ and

$$
\|L v\|_{1} \leq M\|v\| \leq M\|v\|_{1}, \quad \forall v \in Y,
$$

where $M=\max \left\{1,(1 / 8)\left(1+\sum_{i=1}^{m-2} \alpha_{i} /\left(1-\sum_{i=1}^{m-2} \alpha_{i} \eta_{i}\right)\right)\right\}$.

Moreover, $u \in C^{4}[0,1]$ is a solution of $B V P(1.1)$ if and only if $v=-u^{\prime \prime}$ is a solution of the operator equation $v=A v$.

Let the function $\Gamma(s)$ be defined by

$$
\Gamma(s)=\cos s-\sum_{i=1}^{m-2} \alpha_{i} \cos \eta_{i} s, \quad s \in \mathbb{R} .
$$

Then we have the following lemma.

Lemma 2.2. (i) For each $k \geq 1, \Gamma(s)$ has exactly one zero $s_{k} \in I_{k}:=((k-1) \pi, k \pi)$, so

$$
s_{1}<s_{2}<\cdots<s_{k} \longrightarrow+\infty \quad(k \longrightarrow+\infty)
$$

(ii) the characteristic value of $L$ is exactly given by $\mu_{k}=s_{k}^{2}, k=1,2, \ldots$, and the eigenfunction $\phi_{k}$ corresponding to $\mu_{k}$ is $\phi_{k}(t)=\cos s_{k} t$;

(iii) the algebraic multiplicity of each characteristic value $\mu_{k}$ of $L$ is 1 ;

(iv) $\phi_{k} \in T_{k}^{+}$for $k=1,2,3, \ldots$, and $\phi_{1}$ is strictly positive on $(0,1)$. 
Proof. From [5] and by a similar analysis as in the proof of [6, Lemma 3.3] we obtain (i) and (ii).

Now we assert (iii) holds. Suppose, on the contrary, there exists $y \in Y$ such that (I$\left.\mu_{k} L\right) y=\mu_{k}^{-1} \phi_{k}$. Then $y \in Z$ and

$$
-y^{\prime \prime}-s_{k}^{2} y=\cos s_{k} t
$$

From $y^{\prime}(0)=0$ we know the general solution of this differential equation is

$$
y=C \cos s_{k} t-\frac{1}{2 s_{k}} t \sin s_{k} t
$$

From (i) and (ii) of this lemma, $C \cos s_{k} t$ satisfies the boundary condition. Thus

$$
\cos s_{k}=\sum_{i=1}^{m-2} \alpha_{i} \cos \eta_{i} s_{k}, \quad \sin s_{k}=\sum_{i=1}^{m-2} \alpha_{i} \eta_{i} \sin \eta_{i} s_{k} .
$$

Then, by (1.2),

$$
\begin{aligned}
1 & =\left(\sum_{i=1}^{m-2} \alpha_{i} \cos \eta_{i} s_{k}\right)^{2}+\left(\sum_{i=1}^{m-2} \alpha_{i} \eta_{i} \sin \eta_{i} s_{k}\right)^{2} \\
& \leq \sum_{i, j=1}^{m-2} \alpha_{i} \alpha_{j}\left(\left|\cos \eta_{i} s_{k} \cos \eta_{j} s_{k}\right|+\left|\sin \eta_{i} s_{k} \sin \eta_{j} s_{k}\right|\right) \\
& \leq\left(\sum_{i=1}^{m-2} \alpha_{i}\right)^{2}<1,
\end{aligned}
$$

a contradiction. Thus the algebraic multiplicity of each characteristic value $\mu_{k}$ of $L$ is 1 .

Finally, from $s_{k} \in((k-1) \pi, k \pi)$ and $s_{1} \in(0, \pi / 2)$, it is easy to see that (iv) holds.

Lemma 2.3. For $d=\left(d_{1}, d_{2}\right) \in \mathbb{R}^{+} \times \mathbb{R}^{+} \backslash\{(0,0)\}$, define a linear operator

$$
L_{d} v(t)=\left(d_{1} L^{2}+d_{2} L\right) v(t), \quad \forall t \in I, v \in Y,
$$

where $L$ is defined as in (2.3). Then the generalized eigenvalues of $L_{d}$ are simple and are given by

$$
0<\lambda_{1}\left(L_{d}\right)<\lambda_{2}\left(L_{d}\right)<\cdots<\lambda_{k}\left(L_{d}\right) \longrightarrow+\infty \quad(k \longrightarrow+\infty),
$$

where

$$
\lambda_{k}\left(L_{d}\right)=\frac{\mu_{k}^{2}}{d_{1}+d_{2} \mu_{k}}
$$


The generalized eigenfunction corresponding to $\lambda_{k}\left(L_{d}\right)$ is

$$
\phi_{k}(t)=\cos s_{k} t
$$

where $\mu_{k}, s_{k}, \phi_{k}$ are as in Lemma 2.2 .

Proof. Suppose there exist $\lambda$ and $v \neq 0$ such that $v=\lambda L_{d} v$. Set $u(t)=L v(t)$. Then from (2.2)(2.7) and (2.15) it is easy to see that $u \neq 0$ and

$$
\begin{gathered}
u^{(4)}(t)=\lambda\left(d_{1} u(t)-d_{2} u^{\prime \prime}(t)\right), \quad t \in(0,1) ; \\
u^{\prime}(0)=0, \quad u(1)=\sum_{i=1}^{m-2} \alpha_{i} u\left(\eta_{i}\right) \\
u^{\prime \prime \prime}(0)=0, \quad u^{\prime \prime}(1)=\sum_{i=1}^{m-2} \alpha_{i} u^{\prime \prime}\left(\eta_{i}\right) .
\end{gathered}
$$

Denote $L^{-1} u=-u^{\prime \prime}$ for $u \in Z$. Then there exist two complex numbers $r_{1}$ and $r_{2}$ such that

$$
u^{(4)}(t)-\lambda\left(d_{1} u(t)-d_{2} u^{\prime \prime}(t)\right)=\left(L^{-1}-r_{2} I\right)\left(L^{-1}-r_{1} I\right) u(t)=0
$$

Now if there exists some $r_{i}(i=1,2)$ such that

$$
\left(L^{-1}-r_{i} I\right) u(t)=0
$$

then by Lemma 2.2 we know $r_{i}=s_{k}^{2}=\mu_{k}$ for some $k \in \mathbb{N}$, and consequently

$$
u(t)=\cos s_{k} t
$$

is a nontrivial solution. Substituting (2.22) into (2.19), we have

$$
\lambda=\frac{\mu_{k}^{2}}{d_{1}+d_{2} \mu_{k}} .
$$

On the other hand, suppose, for example,

$$
\left(L^{-1}-r_{1} I\right) u(t) \neq 0, \quad\left(L^{-1}-r_{2} I\right)\left(L^{-1}-r_{1} I\right) u(t)=0
$$


Let $w(t):=\left(L^{-1}-r_{1} I\right) u(t)$. Then $\left(L^{-1}-r_{2} I\right) w(t)=0$. Reasoning as previously mentioned, we have $r_{2}=s_{k}^{2}$ for some $k \in \mathbb{N}$, and consequently $w(t)=a \cos s_{k} t(a \neq 0)$ is a nontrivial solution. Therefore,

$$
\left(L^{-1}-r_{1} I\right) u(t)=a \cos s_{k} t
$$

If $r_{1}=s_{k}^{2}$, then the general solution of the differential equation (2.25), satisfying $u^{\prime}(0)=$ 0 , is

$$
u(t)=C \cos s_{k} t-\frac{a}{2 s_{k}} t \sin s_{k} t
$$

which is similar to (2.12). Reasoning as in the proof of Lemma 2.2 we can get a contradiction. Thus $r_{1} \neq s_{k}^{2}$ and the general solution of (2.25), satisfying $u^{\prime}(0)=0$, is

$$
u(t)=\tilde{u}(t)+\frac{a \cos s_{k} t}{s_{k}^{2}-r_{1}}
$$

where $\tilde{u}(t)$ is the general solution of homogeneous differential equation corresponding to $(2.25)$

$$
\left(L^{-1}-r_{1} I\right) u(t)=0
$$

Notice the term $a \cos s_{k} t /\left(s_{k}^{2}-r_{1}\right)$ in (2.27) satisfies the boundary condition of (1.1) at $t=1$, so $\tilde{u}(t)$ also satisfies

$$
\tilde{u}^{\prime}(0)=0, \quad \tilde{u}(1)=\sum_{i=1}^{m-2} \alpha_{i} \tilde{u}\left(\eta_{i}\right)
$$

Therefore, by Lemma 2.2 we know $\tilde{u}(t)=C \cos s_{j} t$ for some $j \in \mathbb{N}$, and consequently

$$
r_{1}=s_{j}^{2} \neq s_{k^{\prime}}^{2} \quad u(t)=C \cos s_{j} t+\frac{a \cos s_{k} t}{s_{k}^{2}-s_{j}^{2}} .
$$

By substituting this into (2.19), we have

$$
a \lambda\left(d_{1}+d_{2} \mu_{k}\right)=a \mu_{k}^{2}, \quad C \lambda\left(d_{1}+d_{2} \mu_{j}\right)=C \mu_{j}^{2} .
$$

Since $\mu_{j} \neq \mu_{k}$, if there exists some $\lambda$ such that (2.31) holds, then

$$
\frac{d_{1}+d_{2} \mu_{k}}{d_{1}+d_{2} \mu_{j}}=\frac{\mu_{k}^{2}}{\mu_{j}^{2}}
$$


which implies

$$
d_{1} d_{2} \neq 0, \quad d_{1}\left(\frac{1}{\mu_{k}}+\frac{1}{\mu_{j}}\right)=-d_{2},
$$

a contradiction with $d_{1}>0$ and $d_{2}>0$.

Consequently, (2.24) does not hold. This together with (2.20)-(2.23) and Lemma 2.2 guarantee that the generalized eigenvalues of $L_{d}$ are given by

$$
0<\lambda_{1}\left(L_{d}\right)<\lambda_{2}\left(L_{d}\right)<\cdots<\lambda_{k}\left(L_{d}\right) \longrightarrow+\infty \quad(k \longrightarrow+\infty),
$$

where $\lambda_{k}\left(L_{d}\right)=\mu_{k}^{2} /\left(d_{1}+d_{2} \mu_{k}\right)$. The generalized eigenfunction corresponding to $\lambda_{k}\left(L_{d}\right)$ is $\phi_{k}(t)=\cos s_{k} t$.

Now we are in a position to show the generalized eigenvalues of $L_{d}$ are simple.

Clearly, from above we know for $\lambda_{k}:=\lambda_{k}\left(L_{d}\right),\left(I-\lambda_{k} L_{d}\right) \phi_{k}=0$ and $\operatorname{dim} N\left(I-\lambda_{k} L_{d}\right)=1$. Suppose there exists an $v \in C^{2}$ such that

$$
\left(I-\lambda_{k} L_{d}\right) v=\frac{1}{\mu_{k}} \phi_{k}(t)
$$

This together with (2.3) and (2.15) guarantee that $v \in Y$. If we let $u(t)=(L v)(t)$ as above, then we have

$$
\begin{gathered}
u^{(4)}(t)-\lambda_{k}\left(d_{1} u(t)-d_{2} u^{\prime \prime}(t)\right)=\cos s_{k} t, \quad t \in(0,1), \\
u^{\prime}(0)=0, \quad u(1)=\sum_{i=1}^{m-2} \alpha_{i} u\left(\eta_{i}\right) ; \quad u^{\prime \prime \prime}(0)=0, \quad u^{\prime \prime}(1)=\sum_{i=1}^{m-2} \alpha_{i} u^{\prime \prime}\left(\eta_{i}\right) .
\end{gathered}
$$

Consider the following homogeneous equation corresponding to (2.36):

$$
u^{(4)}(t)-\frac{\mu_{k}^{2}}{d_{1}+d_{2} \mu_{k}}\left(d_{1} u(t)-d_{2} u^{\prime \prime}(t)\right)=0
$$

The characteristic equation associated with (2.38) is

$$
\lambda^{4}-\frac{\mu_{k}^{2}}{d_{1}+d_{2} \mu_{k}}\left(d_{1}-d_{2} \lambda^{2}\right)=0
$$

Then there exists a real number $\eta$ such that

$$
\left(\lambda^{2}+\mu_{k}\right)\left(\lambda^{2}-\eta\right)=\lambda^{4}-\frac{\mu_{k}^{2}}{d_{1}+d_{2} \mu_{k}}\left(d_{1}-d_{2} \lambda^{2}\right)=0
$$

Notice that $-\eta \mu_{k}=-d_{1} \mu_{k}^{2} /\left(d_{1}+d_{2} \mu_{k}\right)<0$ if $d_{1}>0$. So $\eta>0$ if $d_{1}>0$, and $\eta=0$ if $d_{1}=0$. 
First we consider the case $d_{1}>0$. In this case the general solution of (2.38) is

$$
c_{1} e^{\sqrt{\eta} t}+c_{2} e^{-\sqrt{\eta} t}+c_{3} \cos s_{k} t+c_{4} \sin s_{k} t
$$

After computation we obtain that the general solution of (2.36) is

$$
u(t)=c_{1} e^{\sqrt{\eta} t}+c_{2} e^{-\sqrt{\eta} t}+c_{3} \cos s_{k} t+c_{4} \sin s_{k} t+a t \sin s_{k} t
$$

where $a=-\left(d_{1}+d_{2} \mu_{k}\right) / 2 s_{k}\left(2 d_{1} \mu_{k}+d_{2} \mu_{k}^{2}\right)$. From boundary condition $u^{\prime}(0)=u^{\prime \prime \prime}(0)=0$ in (2.37) it follows that

$$
\begin{gathered}
\sqrt{\eta}\left(c_{1}-c_{2}\right) s_{k} c_{4}=0 ; \\
\eta \sqrt{\eta}\left(c_{1}-c_{2}\right)-s_{k}^{3} c_{4}=0 .
\end{gathered}
$$

By $\eta>0$ and $\mu_{k}>0$, we know $c_{1}-c_{2}=0$ and $c_{4}=0$. Then (2.42) can be rewritten as

$$
u(t)=c_{1}\left[e^{\sqrt{\eta} t}+e^{-\sqrt{\eta} t}\right]+c_{3} \cos s_{k} t+a t \sin s_{k} t .
$$

Notice that the term $c_{3} \cos s_{k} t$ satisfies (2.37). From the boundary condition

$$
\begin{gathered}
u(1)=\sum_{i=1}^{m-2} \alpha_{i} u\left(\eta_{i}\right), \quad u^{\prime \prime}(1)=\sum_{i=1}^{m-2} \alpha_{i} u^{\prime \prime}\left(\eta_{i}\right), \\
\left(t \sin s_{k} t\right)^{\prime \prime}=2 s_{k} \cos s_{k} t-s_{k}^{2} t \sin s_{k} t
\end{gathered}
$$

we have

$$
\begin{array}{r}
c_{1}\left[e^{\sqrt{\eta}}+e^{-\sqrt{\eta}}\right]+a \sin s_{k}=\sum_{i=1}^{m-2} \alpha_{i}\left[c_{1}\left(e^{\sqrt{\eta} \eta_{i}}+e^{-\sqrt{\eta} \eta_{i}}\right)+a \eta_{i} \sin s_{k} \eta_{i}\right] \\
c_{1} \eta\left[e^{\sqrt{\eta}}+e^{-\sqrt{\eta}}\right]-a s_{k}^{2} \sin s_{k}=\sum_{i=1}^{m-2} \alpha_{i}\left[c_{1} \eta\left(e^{\sqrt{\eta} \eta_{i}}+e^{-\sqrt{\eta} \eta_{i}}\right)-a \eta_{i} s_{k}^{2} \sin s_{k} \eta_{i}\right] .
\end{array}
$$

Multiply (2.46) by $s_{k}^{2}$ and then add to (2.47) to obtain

$$
c_{1}\left(\eta+s_{k}^{2}\right)\left[e^{\sqrt{\eta}}+e^{-\sqrt{\eta}}\right]=c_{1}\left(\eta+s_{k}^{2}\right) \sum_{i=1}^{m-2} \alpha_{i}\left(e^{\sqrt{\eta} \eta_{i}}+e^{-\sqrt{\eta} \eta_{i}}\right) .
$$

On the other hand, from (1.2) it can be seen that

$$
e^{\sqrt{\eta}}+e^{-\sqrt{\eta}}>\sum_{i=1}^{m-2} \alpha_{i}\left(e^{\sqrt{\eta} \eta_{i}}+e^{-\sqrt{\eta} \eta_{i}}\right)
$$


This together with (2.48) guarantee that $c_{1}=0$. Therefore, (2.42) reduces to

$$
u(t)=c_{3} \cos s_{k} t+a t \sin s_{k} t .
$$

Similar to (2.12), a contradiction can be derived.

Next consider the case $d_{1}=0$. Then $\eta=0$ from above. In this case the general solution of $(2.38)$ is

$$
c_{1}+c_{2} t+c_{3} \cos s_{k} t+c_{4} \sin s_{k} t
$$

By a similar process, one can easily get a contradiction.

To sum up, the generalized eigenvalues of $L_{d}$ are simple, and the proof of this lemma is complete.

\section{Main Results}

We now list the following hypotheses for convenience.

(H1) There exists $a=\left(a_{1}, a_{2}\right) \in \mathbb{R}^{+} \times \mathbb{R}^{+} \backslash\{(0,0)\}$ such that

$$
f(x, y)=a_{1} x-a_{2} y+o(|(x, y)|), \quad \text { as }|(x, y)| \longrightarrow 0,
$$

where $(x, y) \in \mathbb{R} \times \mathbb{R}$, and $|(x, y)|:=\max \{|x|,|y|\}$.

(H2) There exists $b=\left(b_{1}, b_{2}\right) \in \mathbb{R}^{+} \times \mathbb{R}^{+} \backslash\{(0,0)\}$ such that

$$
f(x, y)=b_{1} x-b_{2} y+o(|(x, y)|), \quad \text { as }|(x, y)| \longrightarrow \infty
$$

(H3) There exists $R>0$ such that

$$
|f(x, y)|<\frac{R}{M}, \quad \text { for }(x, y) \in\{(x, y):|x| \leq M R,|y| \leq R\}
$$

where $M$ is defined as in Lemma 2.1.

(H4) There exist two constants $r_{1}<0<r_{2}$ such that $f\left(x,-r_{1}\right) \geq 0$ and $f\left(x,-r_{2}\right) \leq 0$ for $x \in[-M r, M r]$, and $f(x,-y)$ satisfies a Lipschitz condition in $y$ for $(x, y) \in$ $[-M r, M r] \times\left[r_{1}, r_{2}\right]$, where $r=\max \left\{\left|r_{1}\right|, r_{2}\right\}$.

Now we are ready to give our main results.

Theorem 3.1. Suppose (H1)-(H2) hold. Suppose there exists two integers $i_{0} \geq 0$ and $k>0$ such that either

$$
\frac{\mu_{i_{0}+k}^{2}}{a_{1}+a_{2} \mu_{i_{0}+k}}<1<\frac{\mu_{i_{0}+1}^{2}}{b_{1}+b_{2} \mu_{i_{0}+1}}
$$


or

$$
\frac{\mu_{i_{0}+k}^{2}}{b_{1}+b_{2} \mu_{i_{0}+k}}<1<\frac{\mu_{i_{0}+1}^{2}}{a_{1}+a_{2} \mu_{i_{0}+1}}
$$

holds. Then BVP (1.1) has at least $2 k$ nontrivial solutions.

Theorem 3.2. Suppose (H1) and (H2) hold and one of (H3) and (H4) hold. Suppose there exists two integers $i_{0}$ and $j_{0}$ such that

$$
\frac{\mu_{i_{0}}^{2}}{a_{1}+a_{2} \mu_{i_{0}}}<1, \quad \frac{\mu_{j_{0}}^{2}}{b_{1}+b_{2} \mu_{j_{0}}}<1 .
$$

Then BVP(1.1) has at least $2\left(i_{0}+j_{0}\right)$ solutions.

To set it up we first consider global results for the equation

$$
v=\lambda A v,
$$

on $Y$, where $\lambda \in \mathbb{R}$, and the operator $A$ is defined as in (2.7). Under the condition (H1), (3.1 $)$ can be rewritten as

$$
v=\lambda L_{a} v+H_{a}(\lambda, v)
$$

here $H_{a}(\lambda, v)=\lambda A v-\lambda L_{a} v, L_{a}$ is defined as in (2.12) (replacing $d$ with $a$ ). Obviously, by (H1) and Lemma 2.1-2.3, it can be seen that $H_{a}(\lambda, v)$ is $o\left(\|v\|_{1}\right)$ for $v$ near 0 uniformly on bounded $\lambda$ intervals and $L_{a}$ is a compact linear map on $Y$. A solution of $\left(3.1_{\lambda}\right)$ is a pair $(\lambda, v) \in E$. By (H1), the known curve of solutions $\{(\lambda, 0) \mid \lambda \in \mathbb{R}\}$ will henceforth be referred to as the trivial solutions. The closure of the set on nontrivial solutions of $\left(3.1_{\curlywedge}\right)$ will be denoted by $\Sigma$ as in Lemma 1.1.

If $H_{a}(\lambda, v) \equiv 0$, then (3.7) becomes a linear system

$$
v=\lambda L_{a} v
$$

By Lemma 2.3, (3.8) possesses an increasing sequence of simple eigenvalues

$$
0<\lambda_{1}<\lambda_{2}<\cdots<\lambda_{k}<\cdots \text {, with } \quad \lambda_{k}=\frac{\mu_{k}^{2}}{a_{1}+a_{2} \mu_{k}} \quad \text { as } k \longrightarrow+\infty
$$

Any eigenfunction $\phi_{k}=\cos s_{k} t$ corresponding to $\lambda_{k}$ is in $T_{k}^{+}$.

A similar analysis as in [6, Proposition 4.1] yields the following results.

Lemma 3.3. Suppose that $(\lambda, v)$ is a solution of $\left(3.1_{\lambda}\right)$ and $v \not \equiv 0$. Then $v \in \cup_{i=1}^{\infty} T_{i}$. 
Lemma 3.4. Assume that (H1) holds and $\lambda_{k}$ is defined by (3.9). Then for each integer $k>0$ and each $v=+$, or - , there exists a continua $C_{k}^{v}$ of solutions of $\left(3.1_{\curlywedge}\right)$ in $\Phi_{k}^{v} \cup\left\{\left(\lambda_{k}, 0\right)\right\}$, which meets $\left\{\left(\lambda_{k}, 0\right)\right\}$ and $\infty$ in $\Sigma$.

Proof. Consider (3.7) as a bifurcation problem from the trivial solution. From Lemma 1.1 and condition (H1) it follows that for each positive integer $k \in \mathbb{N}, \Sigma$ contains a component $C_{k} \subseteq E=\mathbb{R} \times Y$ which can be decomposed into two subcontinua $C_{k^{\prime}}^{+}, C_{k}^{-}$such that for some neighborhood $B$ of $\left(\lambda_{k}, 0\right)$,

$$
(\lambda, v) \in C_{k}^{+}\left(C_{k}^{-}\right) \cap B, \quad(\lambda, v) \neq\left(\lambda_{k}, 0\right)
$$

$\operatorname{imply}(\lambda, v)=\left(\lambda, \alpha \phi_{k}+w\right)$, where $\alpha>0(\alpha<0)$ and $\left|\lambda-\lambda_{k}\right|=o(1),\|w\|_{1}=o(|\alpha|)$ at $\alpha=0$.

By (3.7) and the continuity of the operator $A: Y \rightarrow Z$, the set $C_{k}^{v}$ lies in $\mathbb{R} \times Z$ and the injection $C_{k}^{v} \rightarrow \mathbb{R} \times Z$ is continuous. Thus, $C_{k}^{v}$ is also a continuum in $\mathbb{R} \times Z$, and the above properties hold in $\mathbb{R} \times Z$.

Since $T_{k}$ is open in $Z$ and $\phi_{k} \in T_{k}^{+}$, we know

$$
\frac{v}{\alpha}=\phi_{k}+\frac{w}{\alpha} \in T_{k}^{+}
$$

for $0 \neq \alpha$ sufficiently small. Then there exists $\varepsilon_{0}>0$ such that for $\varepsilon \in\left(0, \varepsilon_{0}\right)$, we have

$$
(\lambda, v) \in \Phi_{k}, \quad\left(\frac{C_{k}}{\left\{\left(\lambda_{k}, 0\right)\right\}}\right) \cap B_{\varepsilon} \subset \Phi_{k}
$$

where $B_{\varepsilon}$ is an open ball in $\mathbb{R} \times Z$ of radius $\varepsilon$ centered at $\left(\lambda_{k}, 0\right)$. It follows from the proof of [6, Proposition 4.1] that

$$
(\lambda, v) \in C_{k} \cap\left(\mathbb{R} \times \partial T_{k}\right) \Longrightarrow u=0,
$$

which means $C_{k} \backslash\left\{\left(\lambda_{k}, 0\right)\right\} \cap \partial \Phi_{k}=\emptyset$. Consequently, $C_{k}$ lies in $\Phi_{k} \cup\left\{\left(\lambda_{k}, 0\right)\right\}$.

Similarly we have that $C_{k}^{v}$ lies in $\Phi_{k}^{v} \cup\left\{\left(\lambda_{k}, 0\right)\right\}(v=+$ or -$)$.

Next we show alternative (ii) of Lemma 1.1 is impossible. If not, without loss of generality, assume that $C_{k}^{+}$meets $\left(\lambda_{i}, 0\right)$ with $\lambda_{k} \neq \lambda_{i} \in \sigma\left(L_{a}\right)$. Then there exists a sequence $\left(\xi_{m}, z_{m}\right) \in C_{k}^{+}$with $\xi_{m} \rightarrow \lambda_{i}$ and $z_{m} \rightarrow 0$ as $m \rightarrow+\infty$. Notice that

$$
z_{m}=\xi_{m} L_{a} z_{m}+H\left(\xi_{m}, z_{m}\right)
$$

Dividing this equation by $\left\|z_{m}\right\|_{1}$ and using Lemma 2.1 and $H\left(\xi_{m}, z_{m}\right)=o\left(\left\|z_{m}\right\|_{1}\right)$ as $m \rightarrow$ $+\infty$, we may assume without loss of generality that $z_{m} /\left\|z_{m}\right\|_{1} \rightarrow \bar{z}$ as $m \rightarrow+\infty$. Thus from (3.14) it follows that

$$
\bar{z}=\lambda_{i} L_{a} \bar{z} .
$$

Since $\bar{z} \neq 0$, by Lemmas 2.2 and $2.3, \bar{z}$ belongs to $T_{i}^{+}$or $T_{i}^{-}$. By $\left(3.1_{\curlywedge}\right)$ and the continuity of the operator $A: Y \rightarrow Z$, from $\left\|z_{m}-\bar{z}\right\|_{1} \rightarrow 0$ it follows that $\left\|z_{m}-\bar{z}\right\|_{2} \rightarrow 0$. Notice that $T_{i}^{+}$and 
$T_{i}^{-}$are open in $Z$. Therefore, $z_{m} \in T_{i}^{+}$or $T_{i}^{-}$for $m$ sufficiently large, which is a contradiction with $z_{m} \in T_{k}^{+}(m \geq 1), i \neq k$. Hence alternative (ii) of Lemma 1.1 is not possible.

Finally it remains to show alternative (iii) of Lemma 1.1 is impossible. In fact, notice that $T_{k}^{-}=-T_{k}^{+}$, and $T_{k}^{-} \cap T_{k}^{+}=\emptyset$. If $u \in T_{k}^{+}$, then $-u \in T_{k}^{-}$. This guarantees that $C_{k}^{v}$ does not contain a pair of points $(\lambda, v),(\lambda,-v), v \neq 0$.

Therefore alternative (i) of Lemma 1.1 holds. This implies that for each integer $k \in \mathbb{N}$ and each $v=+$, or - , there exists a continua $C_{k}^{v}$ of solutions of $\left(3.1_{\lambda}\right)$ in $\Phi_{k}^{v} \cup\left\{\left(\lambda_{k}, 0\right)\right\}$, which meets $\left\{\left(\lambda_{k}, 0\right)\right\}$ and $\infty$ in $\Sigma$.

Under the condition $(\mathrm{H} 2),\left(3.1_{\curlywedge}\right)$ can be rewritten as

$$
v=\lambda L_{b} v+H_{b}(\lambda, v)
$$

here $H_{b}(\lambda, v)=\lambda A v-\lambda L_{b} v, L_{b}$ is defined as in (2.12) (replacing $d$ with $b$ ).

Let $h(x, y):=f(x, y)-b_{1} x+b_{2} y$. Then from (H2) it follows that $\lim _{|(x, y)| \rightarrow \infty} h(x, y) /$ $|(x, y)|=0$. Define a function

$$
\widehat{h}(r):=\max \{|h(x, y)|:|(x, y)| \leq r\} .
$$

Then $\widehat{h}(r)$ is nondecreasing and

$$
\lim _{r \rightarrow \infty} \frac{\widehat{h}(r)}{r}=0 .
$$

Obviously, by (3.18) and Lemma 2.1, it can be seen that $H_{b}(\lambda, v)$ is $o\left(\|v\|_{1}\right)$ for $v$ near $\infty$ uniformly on bounded $\lambda$ intervals and $L_{b}$ is a compact linear map on $Y$.

Similar to (3.8), by Lemma 2.3, $L_{b}$ possesses an increasing sequence of simple eigenvalues

$$
0<\bar{\lambda}_{1}<\bar{\lambda}_{2}<\cdots<\bar{\lambda}_{k}<\cdots \text {, with } \quad \bar{\lambda}_{k}=\frac{\mu_{k}^{2}}{b_{1}+b_{2} \mu_{k}} \quad \text { as } k \longrightarrow+\infty \text {. }
$$

Note $\phi_{k}=\cos s_{k} t$ is an eigenfunction corresponding to $\bar{\lambda}_{k}$. Obviously, it is in $T_{k}^{+}$.

Lemma 3.5. Assume that (H1)-(H2) holds. Then for each integer $k>0$ and each $v=+$, or - , there exists a continua $D_{k}^{v}$ of $\Sigma$ in $\Phi_{k}^{v} \cup\left\{\left(\bar{\lambda}_{k}, \infty\right)\right\}$ coming from $\left\{\left(\bar{\lambda}_{k}, \infty\right)\right\}$, which meets $\left(\bar{\lambda}_{k}, 0\right)$ or has an unbounded projection on $\mathbb{R}$.

Proof. From (2.7), (3.16), and (3.18) it follows that $H_{b}(\lambda, v)$ is continuous on $E, H_{b}(\lambda, v)=$ $o\left(\|v\|_{1}\right)$ at $v=\infty$ uniformly on bounded $\lambda$ intervals. Moreover, as in the proof of [12, Theorem 2.4], one can see that $\|v\|_{1}^{2} H_{b}\left(\lambda, v /\|v\|_{1}^{2}\right)$ is compact. From Lemma 2.3 we know $\bar{\lambda}_{k}$ is a simple characteristic value of $L_{b}$ for each integer $k \in \mathbb{N}$. Thus by Lemmas 1.2 and $1.3, \Sigma$ contains a component $D_{k}$ which can be decomposed into two subcontinua $D_{k^{\prime}}^{+} D_{k}^{-}$which meet $\left\{\left(\bar{\lambda}_{k}, \infty\right)\right\}$.

Now we show that for a smaller neighborhood $\mathfrak{I} \subset \mathfrak{l}$ of $\left(\bar{\lambda}_{k}, \infty\right),(\lambda, v) \in D_{k}^{+}\left(D_{k}^{-}\right) \cap \mathfrak{I}$ and $(\lambda, v) \neq\left(\bar{\lambda}_{k}, \infty\right)$ imply that $v \in T_{k}^{+}\left(T_{k}^{-}\right)$. In fact, by Lemma 1.3 we already know that there 
exists a neighborhood $\mathfrak{I} \subset \mathfrak{\wp}$ of $\left(\bar{\lambda}_{k}, \infty\right)$ satisfying $(\lambda, v) \in D_{k}^{+}\left(D_{k}^{-}\right) \cap \mathfrak{I}$ and $(\lambda, v) \neq\left(\bar{\lambda}_{k}, \infty\right)$ imply $(\lambda, v)=\left(\lambda, \alpha v_{k}+w\right)$ where $\alpha>0(\alpha<0)$ and $\left|\lambda-\bar{\lambda}_{k}\right|=o(1),\|w\|_{1}=o(|\alpha|)$ at $|\alpha|=\infty$.

As in the proof of Lemma 3.4, $D_{k}^{v}$ is also a continuum in $\mathbb{R} \times Z$, and the above properties hold in $\mathbb{R} \times Z$. Since $T_{k}^{v}$ is open in $Z$ and $w / \alpha$ is smaller compared to $\phi_{k} \in T_{k}^{+}$near $\alpha=+\infty$, $\phi_{k}+w / \alpha$ and therefore $v=\alpha \phi_{k}+w \in T_{k}^{+}$for $\alpha$ near $+\infty$. Here and in the following the same argument works if + is replaced by - .

Therefore, $D_{k}^{+} \cap \mathfrak{I} \subset\left(\mathbb{R} \times T_{k}^{+}\right) \cup\left(\bar{\lambda}_{k}, \infty\right)$. Now we have two cases to consider, that is, $D_{k}^{+} \backslash \mathfrak{I}$ is bounded or unbounded. First suppose $D_{k}^{+} \backslash \mathfrak{I}$ is bounded. Then there exists $(\lambda, v) \in \partial D_{k}^{+}$ with $v \in \partial T_{k}^{+}$. If $v \neq 0$, by Lemma 3.3 we know $v \in T_{j}^{v}$ for some positive integer $j \neq k$ and $v \in\{+,-\}$. As in the proof of Lemma 3.4, we get a contradiction, which means $v=0$. Thus there exists a sequence $\left(\xi_{m}, z_{m}\right) \in D_{k}^{+}$with $z_{m} \rightarrow v \equiv 0$ as $m \rightarrow+\infty$. This together with (H1) guarantee that $\left(\xi_{m}, z_{m}\right)$ satisfies (3.14).

As in the proof of Lemma 3.4, we may assume without loss of generality that $z_{m} /\left\|z_{m}\right\|_{1} \rightarrow \bar{z}$ and $\xi_{m} \rightarrow \xi$ as $m \rightarrow+\infty$. Then we have

$$
\bar{z}=\xi L_{a} \bar{z} .
$$

Since $\bar{z} \neq 0, \xi \neq 0$ is an eigenvalue of operator $L_{a}$. From this, (3.9), and (3.19) it follows that $\xi=\lambda_{j}$ for some positive integer $j$. Then by Lemma $2.3, \bar{z}$ belongs to $T_{j}^{+}$or $T_{j}^{-}$. Notice that $\left\|z_{m}-\bar{z}\right\|_{1} \rightarrow 0$ and so $\left\|z_{m}-\bar{z}\right\|_{2} \rightarrow 0$ as in the proof of Lemma 3.4. Thus $z_{m} \in T_{j}^{+}$or $T_{j}^{-}$for $m$ sufficiently large since $T_{j}^{+}$and $T_{j}^{-}$are open. This together with $z_{m} \in T_{k}^{+}(m \geq 1)$ guarantee that $k=j$. This means $D_{k}^{+}$meets $\left(\lambda_{k}, 0\right)$ if $D_{k}^{+} \backslash \mathfrak{I}$ is bounded.

Next suppose $D_{k}^{+} \backslash \mathfrak{I}$ is unbounded. In this case we show $D_{k}^{+} \backslash \mathfrak{I}$ has an unbounded projection on $\mathbb{R}$. If not, then there exists a sequence $\left(\zeta_{m}, y_{m}\right) \in D_{k}^{+} \backslash \mathfrak{I}$ with $\zeta_{m} \rightarrow \bar{\zeta}$ and $\left\|y_{m}\right\|_{1} \rightarrow+\infty$ as $m \rightarrow+\infty$. Let $x_{m}:=y_{m} /\left\|y_{m}\right\|_{1}, m \geq 1$. From the fact that

$$
y_{m}=\zeta_{m} L_{b} y_{m}+H_{b}\left(\zeta_{m}, y_{m}\right)
$$

it follows that

$$
x_{m}=\zeta_{m} L_{b} x_{m}+\frac{H_{b}\left(\zeta_{m}, y_{m}\right)}{\left\|y_{m}\right\|_{1}}
$$

Notice that $L_{b}: Y \rightarrow Y$ is completely continuous. We may assume that there exists $w \in Y$ with $\|w\|_{1}=1$ such that $\left\|x_{m}-w\right\|_{1} \rightarrow 0$ as $m \rightarrow+\infty$. obtains

Letting $m \rightarrow+\infty$ in (3.22) and noticing $H_{b}\left(\zeta_{m}, y_{m}\right) /\left\|y_{m}\right\|_{1} \rightarrow 0$ as $m \rightarrow+\infty$ one

$$
w=\bar{\zeta} L_{b} w
$$

Since $w \neq 0, \bar{\zeta} \neq 0$ is an eigenvalue of operator $L_{b}$, that is, $\bar{\zeta}=\bar{\lambda}_{k_{0}}$ for some positive integer $k_{0} \neq k$. Then by Lemma $2.3 w$ belongs to $T_{k_{0}}^{+}$or $T_{k_{0}}^{-}$. Notice the fact that $\left\|x_{m}-w\right\|_{1} \rightarrow 0$ and so $\left\|x_{m}-w\right\|_{2} \rightarrow 0$ as in the proof of Lemma 3.4. Thus $x_{m} \in T_{k_{0}}^{+}$or $T_{k_{0}}^{-}$for $m$ sufficiently large since $T_{k_{0}}^{+}$and $T_{k_{0}}^{-}$are open. This is a contradiction with $x_{m} \in T_{k}^{+}(m \geq 1)$. Thus $D_{k}^{+} \backslash \mathfrak{I}$ has an unbounded projection on $\mathbb{R}$. 
Proof of Theorem 3.1. Suppose first that

$$
\frac{\mu_{i_{0}+k}^{2}}{a_{1}+a_{2} \mu_{i_{0}+k}}<1<\frac{\mu_{i_{0}+1}^{2}}{b_{1}+b_{2} \mu_{i_{0}+1}} .
$$

Using the notation of (3.9) and (3.19), this means $\lambda_{i_{0}+k}<1<\bar{\lambda}_{i_{0}+1}$ and so from Lemma 2.3 we know

$$
\lambda_{i_{0}+1}<\lambda_{i_{0}+2}<\cdots<\lambda_{i_{0}+k}<1<\bar{\lambda}_{i_{0}+1}<\bar{\lambda}_{i_{0}+2}<\cdots<\bar{\lambda}_{i_{0}+k}
$$

Consider (3.7) as a bifurcation problem from the trivial solution. We need only show that

$$
C_{i_{0}+j}^{v} \bigcap(\{1\} \times Y) \neq \emptyset, \quad j=1,2, \ldots, k ; v=+,-.
$$

Suppose, on the contrary and without loss of generality, that

$$
C_{i_{0}+i}^{+} \bigcap(\{1\} \times Y)=\emptyset, \quad \text { for some } i, 1 \leq i \leq k .
$$

By Lemma 3.4 we know that $C_{i_{0}+i}^{+}$joins $\left(\lambda_{i_{0}+i}, 0\right)$ to infinity in $\Sigma$ and $(\lambda, v)=(0,0)$ is the unique solution of $\left(3.1_{\lambda}\right)$ (in which $\lambda=0$ ) in $E$. This together with $\lambda_{i_{0}+i}<1$ guarantee that there exists a sequence $\left\{\left(\zeta_{m}, y_{m}\right)\right\} \subset C_{i_{0}+i}^{+}$such that $\zeta_{m} \in(0,1)$ and $\left\|y_{m}\right\|_{1} \rightarrow \infty$ as $m \rightarrow+\infty$. We may assume that $\zeta_{m} \rightarrow \bar{\zeta} \in[0,1]$ as $m \rightarrow+\infty$. Let $x_{m}:=y_{m} /\left\|y_{m}\right\|_{1}, m \geq 1$, then (3.22) holds. Similarly, we may assume that there exists $w \in Y$ with $\|w\|_{1}=1$ such that $\left\|x_{m}-w\right\|_{1} \rightarrow 0$ as $m \rightarrow+\infty$ and (3.23) holds. From the proof of Lemma 3.5 one can see $\bar{\zeta}=\bar{\lambda}_{i_{0}+i}$, which contradicts $\bar{\lambda}_{i_{0}+i}>1$. Thus (3.27) is not true, which means (3.26) holds.

Next suppose that

$$
\frac{\mu_{i_{0}+k}^{2}}{b_{1}+b_{2} \mu_{i_{0}+k}}<1<\frac{\mu_{i_{0}+1}^{2}}{a_{1}+a_{2} \mu_{i_{0}+1}} .
$$

This means

$$
\bar{\lambda}_{i_{0}+1}<\bar{\lambda}_{i_{0}+2}<\cdots<\bar{\lambda}_{i_{0}+k}<1<\lambda_{i_{0}+1}<\lambda_{i_{0}+2}<\cdots<\lambda_{i_{0}+k}
$$

Consider (3.16) as a bifurcation problem from infinity. As above we need only to prove that

$$
D_{i_{0}+j}^{v} \bigcap(\{1\} \times Y) \neq \emptyset, \quad j=1,2, \ldots, k ; v=+,-.
$$

From Lemma 3.5, we know that $D_{i_{0}+j}^{v}$ comes from $\left\{\left(\bar{\lambda}_{i_{0}+j}, \infty\right)\right\}$, meets $\left(\lambda_{i_{0}+j}, 0\right)$ or has an unbounded projection on $\mathbb{R}$. If it meets $\left(\lambda_{i_{0}+j}, 0\right)$, then the connectedness of $D_{i_{0}+j}^{v}$ and $\lambda_{i_{0}+j}>1$ 
guarantees that (3.30) is satisfied. On the other hand, if $D_{i_{0}+j}^{v}$ has an unbounded projection on $\mathbb{R}$, notice that $(\lambda, v)=(0,0)$ is the unique solution of $\left(3.1_{\lambda}\right)$ (in which $\lambda=0$ ) in $E$, so $(3.30)$ also holds.

Proof of Theorem 3.2. First suppose that (H3) holds. Then there exists $\varepsilon>0$ such that

$$
(1+\varepsilon)|f(x, y)|<\frac{R}{M}, \quad \text { for }(x, y) \in\{(x, y):|x| \leq M R,|y| \leq R\} .
$$

Let $(\lambda, v)$ be a solution of $\left(3.1_{\curlywedge}\right)$ such that $0 \leq \lambda<1+\varepsilon$ and $\|v\|_{1} \leq R$. Then by (2.7), $\left(3.1_{\curlywedge}\right),(3.31)$ and Lemma 2.1 it is easy to see

$$
\|v\|_{1}=\lambda\|A v\|_{1}=\lambda\|L F v\|_{1} \leq \lambda M\|F v\|=M \max _{t \in[0,1]}|\lambda f((L v)(t),-v(t))|<M \frac{R}{M}=R .
$$

Therefore,

$$
\Sigma \cap\left([0,1+\varepsilon] \times \partial \bar{B}_{R}\right)=\emptyset
$$

This together with (3.32) and Lemmas 3.4 and 3.5 implies that

$$
\begin{gathered}
C_{k}^{v} \cap\left([0,1+\varepsilon] \times \bar{B}_{R}\right) \subset[0,1+\varepsilon] \times B_{R}, \quad k=1,2, \ldots, i_{0} ; \\
D_{j}^{v} \cap\left([0,1+\varepsilon] \times \partial \bar{B}_{R}\right)=\emptyset, \quad j=1,2, \ldots, j_{0} ;
\end{gathered}
$$

where $B_{R}=\left\{v \in Y \mid\|v\|_{1}<R\right\}$ and $\bar{B}_{R}=\left\{v \in Y \mid\|v\|_{1} \leq R\right\}, C_{k}^{v}$ and $D_{j}^{v}$ are obtained from Lemmas 3.4 and 3.5, respectively.

Since $C_{k}^{v}$ is a unbounded component of solutions of $\left(3.1_{\curlywedge}\right)$ joining $\left(\lambda_{k}, 0\right)$ in $E$, it follows from (3.33) and (3.34) that $C_{k}^{v}$ crosses the hyperplane $\{1\} \times Y$ with $\left(1, v^{v}\right)$ such that $\left\|v^{v}\right\|_{1}<R$ $\left(v=+\right.$ or $\left.-, k=1,2, \ldots, i_{0}\right)$. This means BVP $(2.5)$ has $2 i_{0}$ nontrivial solutions $\left\{v_{i}^{v}\right\}_{1}^{i_{0}}$ in which $v_{1}^{+}$and $v_{1}^{-}$are positive and negative solutions, respectively.

On the other hand, by (3.33), (3.35), and Lemma 3.5 one can obtain

$$
D_{j}^{v} \cap\left(\{1\} \times\left(Y \backslash \bar{B}_{R}\right)\right) \neq \emptyset, \quad j=1,2, \ldots, j_{0} .
$$

This means BVP (2.5) has $2 j_{0}$ nontrivial solutions $\left\{w_{i}^{v}\right\}_{1}^{j_{0}}$ in which $w_{1}^{+}$and $w_{1}^{-}$are positive and negative solutions, respectively.

Now it remains to show this theorem holds when the condition (H4) is satisfied.

From the above we need only to prove that

(i) for $(\lambda, v) \in C_{i}^{v}\left(v=+\right.$ or $\left.-, i=1,2, \ldots, i_{0}\right)$,

$$
r_{1}<v(t)<r_{2}, \quad t \in[0,1] .
$$


(ii) for $(\lambda, v) \in D_{j}^{v}\left(v=+\right.$ or $\left.-, j=1,2, \ldots, j_{0}\right)$, we have that either

$$
\max _{t \in[0,1]} v(t)>r_{2}, \quad t \in[0,1]
$$

or

$$
\min _{t \in[0,1]} v(t)<r_{1}, \quad t \in[0,1]
$$
either

In fact, like in [13], suppose on the contrary that there exists $(\lambda, v) \in C_{i}^{v} \cup D_{j}^{v}$ such that

$$
\max \{v(t): t \in[0,1]\}=r_{2}
$$

or

$$
\min \{v(t): t \in[0,1]\}=r_{1}
$$

for some $i, j$.

First consider the case $\max \{v(t): t \in[0,1]\}=r_{2}$. Then there exists $\bar{t} \in[0,1]$ such that $v(\bar{t})=r_{2}$. Let

$$
\begin{aligned}
& \tau_{0}=: \inf \{t \in[0, \bar{t}]: v(s) \geq \quad \text { for } s \in[t, \bar{t}]\} \\
& \tau_{1}=: \sup \{t \in[\bar{t}, 1]: v(s) \geq 0 \quad \text { for } s \in[\bar{t}, t]\} .
\end{aligned}
$$

Then

$$
\begin{aligned}
& \max \left\{v(t): t \in\left[\tau_{0}, \tau_{1}\right]\right\}=r_{2}, \\
& 0 \leq v(t) \leq r_{2}, \quad t \in\left[\tau_{0}, \tau_{1}\right] .
\end{aligned}
$$

Therefore, $v(t)$ is a solution of the following equation

$$
-v^{\prime \prime}(t)=\lambda f((L v)(t),-v(t)), \quad t \in\left(\tau_{0}, \tau_{1}\right)
$$

with $v\left(\tau_{0}\right)=v\left(\tau_{1}\right)=0$ if $0<\tau_{0}<\tau_{1}<1$ and $v^{\prime}\left(\tau_{0}\right)=0$ if $\tau_{0}=0$.

By (H4), there exists $\bar{M} \geq 0$ such that $f(x,-y)+\bar{M} y$ is strictly increasing in $y$ for $(x, y) \in\left[r_{1}, r_{2}\right] \times[-M r, M r]$, where $r=\max \left\{\left|r_{1}\right|, r_{2}\right\}$. Then

$$
-v^{\prime \prime}+\lambda \bar{M} v=\lambda(f((L v)(t),-v(t))+\bar{M} v), \quad t \in\left(\tau_{0}, \tau_{1}\right)
$$


Using (H4) and Lemma 2.1 again, we can obtain

$$
\begin{aligned}
- & \left(r_{2}-v(t)\right)^{\prime \prime}+\lambda \bar{M}\left(r_{2}-v(t)\right) \\
& =-\lambda\left[f((L v)(t),-v(t))+\bar{M} v(t)-\bar{M} r_{2}\right] \\
& =-\lambda\left[f((L v)(t),-v(t))+\bar{M} v(t)-\left(f\left((L v)(t),-r_{2}\right)+\bar{M} r_{2}\right)\right]-\lambda f\left((L v)(t),-r_{2}\right) \\
& \geq 0, \quad t \in\left(\tau_{0}, \tau_{1}\right)
\end{aligned}
$$

and if $\tau_{1}=1$, by (1.2) we know $v(1)<r_{2}$. Therefore,

$$
\begin{gathered}
r_{2}-v\left(\tau_{0}\right)>0, \quad r_{2}-v\left(\tau_{1}\right)>0 \quad \text { if } 0<\tau_{0}<\tau_{1}<1 ; \\
\left(r_{2}-v\left(\tau_{0}\right)\right)^{\prime}=0 \quad \text { if } \tau_{0}=0 ; \\
r_{2}-v\left(\tau_{1}\right)>0 \quad \text { if } \tau_{1}=1 .
\end{gathered}
$$

This together with (3.47) and the maximum principle [14] imply that $r_{2}-v(t)>0$ in $\left[\tau_{0}, \tau_{1}\right]$, which contradicts (3.43).

The proof in the case $\min \{v(t): t \in[0,1]\}=r_{1}$ is similar, so we omit it.

\section{Acknowledgments}

The Project Supported by NNSF of P. R. China (10871120), the Key Project of Chinese Ministry of Education (No: 209072), and the Science \& Technology Development Funds of Shandong Education Committee (J08LI10).

\section{References}

[1] W. Feng and J. R. L. Webb, "Solvability of $m$-point boundary value problems with nonlinear growth," Journal of Mathematical Analysis and Applications, vol. 212, no. 2, pp. 467-480, 1997.

[2] C. P. Gupta, "A generalized multi-point boundary value problem for second order ordinary differential equations," Applied Mathematics and Computation, vol. 89, no. 1-3, pp. 133-146, 1998.

[3] R. Ma and N. Castaneda, "Existence of solutions of nonlinear m-point boundary-value problems," Journal of Mathematical Analysis and Applications, vol. 256, no. 2, pp. 556-567, 2001.

[4] R. Ma and D. O'Regan, "Nodal solutions for second-order $m$-point boundary value problems with nonlinearities across several eigenvalues," Nonlinear Analysis: Theory, Methods \& Applications, vol. 64, no. 7, pp. 1562-1577, 2006.

[5] R. Ma, "Nodal solutions for a second-order $m$-point boundary value problem," Czechoslovak Mathematical Journal, vol. 56(131), no. 4, pp. 1243-1263, 2006.

[6] B. P. Rynne, "Spectral properties and nodal solutions for second-order, $m$-point, boundary value problems," Nonlinear Analysis: Theory, Methods \& Applications, vol. 67, no. 12, pp. 3318-3327, 2007.

[7] J. Sun, X. Xu, and D. O'Regan, "Nodal solutions for $m$-point boundary value problems using bifurcation methods," Nonlinear Analysis: Theory, Methods \& Applications, vol. 68, no. 10, pp. 3034 3046, 2008.

[8] J. R. L. Webb, "Positive solutions of some three point boundary value problems via fixed point index theory," Nonlinear Analysis: Theory, Methods \& Applications, vol. 47, no. 7, pp. 4319-4332, 2001.

[9] X. Xu, "Multiple sign-changing solutions for some $m$-point boundary-value problems," Electronic Journal of Differential Equations, vol. 2004, no. 89, pp. 1-14, 2004. 
[10] Z. Wei and C. Pang, "Multiple sign-changing solutions for fourth order $m$-point boundary value problems," Nonlinear Analysis: Theory, Methods E Applications, vol. 66, no. 4, pp. 839-855, 2007.

[11] P. H. Rabinowitz, "Some global results for nonlinear eigenvalue problems," Journal of Functional Analysis, vol. 7, no. 3, pp. 487-513, 1971.

[12] P. H. Rabinowitz, "On bifurcation from infinity," Journal of Differential Equations, vol. 14, no. 3, pp. 462-475, 1973.

[13] R. Ma, "Global behavior of the components of nodal solutions of asymptotically linear eigenvalue problems," Applied Mathematics Letters, vol. 21, no. 7, pp. 754-760, 2008.

[14] M. H. Protter and H. F. Weinberger, Maximum Principles in Differential Equations, Springer, New York, NY, USA, 1984. 